\title{
A COMPACT 6D MUON COOLING RING
}

\author{
H.G Kirk*, S.A Kahn, BNL, Upton, NY 11973, USA \\ F.E Mills, FNAL, Batavia, IL 60510, USA \\ D. Cline, A.A Garren, UCLA, CA 90095, USA
}

\section{Abstract}

We discuss a conceptual design for a compact muon cooling system based on a weak-focusing ring loaded with high-pressure hydrogen gas. We demonstrate that such a ring will be capable of cooling a circulating muon beam in each of the three spatial dimensions so that $6 \mathrm{D}$ cooling of the muon beam phase space can be achieved.

\section{INTRODUCTION}

Intense proton sources are being planned as part of a worldwide demand for the development of new intense secondary particle beams. As part of this effort, much research and development has been devoted to creating concepts for the production of intense muon beams[1],[2]. In the case of muons, it may not be sufficient to merely develop an intense source. A method to efficiently capture and reduce the phase space of the beam is required. This is particularly true for such advanced concepts as muon colliders[3] or, to some lesser degree, a neutrino factory based on directing neutrino beams which are generated as decay products of muons circulating in a storage ring[4]. We report here on recent work which could provide a useful technique toward reducing the $6 \mathrm{D}$ phase space of a captured muon beam. Our approach utilizes compact rings consisting of weakly focusing dipole magnets along with the inclusion of high-pressure hydrogen gas which serves as the energyloss absorber and rf cavities which restore the energy of the muon beam.

The ring consists of a number of identical sector-shaped, zero-gradient magnets. Transverse focusing is provided by the magnet edges. The resistance of the gas on the particles and acceleration by the rf cavities provide ionization cooling[5]. The ring has positive dispersion, which means that higher momentum particles have longer paths around the ring (Fig. 1), and thus lose more energy per turn, than the low energy particles do. Consequently, cooling takes place in the longitudinal as well as the transverse dimensions.

Studies of rf cavities operating while filled with highpressure gases, including hydrogen, are on going and encouraging [6]. We chose hydrogen as the loaded gas in order to minimize disruption of the emittance of the circulating muon beam due to multiple scattering and energy straggling.

\footnotetext{
*hkirk@bnl.gov
}

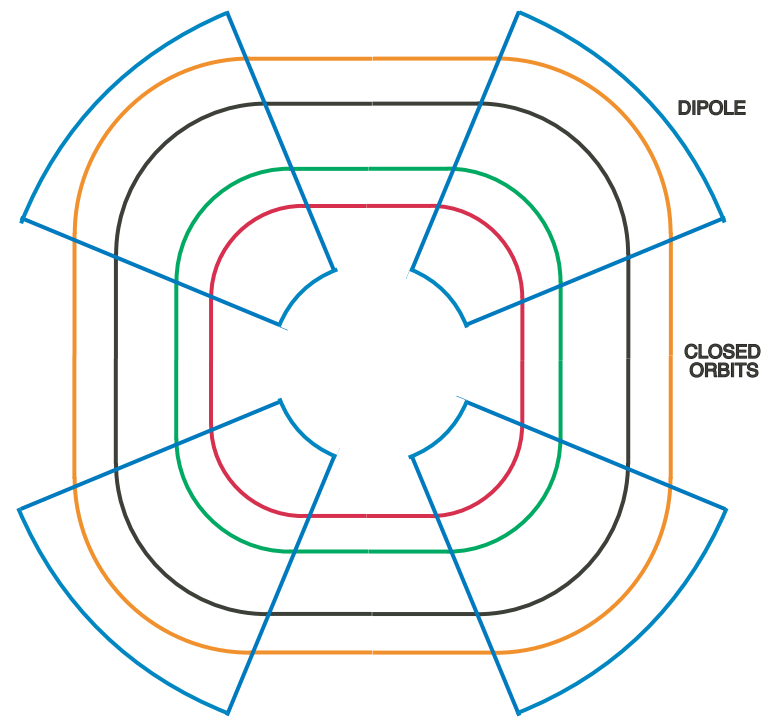

Figure 1: Closed orbits for various beam momenta. Note that positive dispersion results in greater path lengths for higher energy particles.

\section{BEAM DYNAMICS SIMULATIONS}

In general, our approach has been to obtain linear lattice solutions using the code SYNCH [7] and then transferring the lattice parameters to the code ICOOL [8] where the effects of energy loss in absorbers and the subsequent energy recovery in rf cavities can be modeled. Details of our approach to simulations have been previous published [9],[10].

In order to insure cooling in the transverse dimensions, we must place the energy reducing absorbing gas in regions of low beta. Since we intend to fill the entire ring with absorbing gas, this requires us to develop lattices with low beta functions throughout the cell. In our designs, the beam beta functions are always less that $1 \mathrm{~m}$.

We have simulated several lattices in search of an optimal layout, which could yield substantial cooling in all three spatial dimensions. We define as a figure the merit the quantity

$$
\text { Merit }=\text { Transmission } \times \frac{\epsilon_{x_{i}}}{\epsilon_{x_{f}}} \frac{\epsilon_{y_{i}}}{\epsilon_{y_{f}}} \frac{\epsilon_{y_{i}}}{\epsilon_{y_{f}}}
$$

We find that excellent performances, as measured by this merit factor, can be achieved[11]. Merit factors as high as 
400 have been simulated. These solutions require aggressive parameters such as high field dipoles (5T), high operating pressures (100 bar), and high rf accelerating gradients (45 MV/m).

In order to build a low cost cooling ring while still achieving a measurable reduction in the $6 \mathrm{D}$ emittance, we confine ourselves to a peak dipole bending field of $1.8 \mathrm{~T}$, a peak RF gradient of $\leq 15 \mathrm{MV} / \mathrm{m}$ and a peak gas pressure of 10 atmospheres (at $77 \mathrm{deg} \mathrm{K}$ ). With these restricted parameters we have still been able to achieve a merit factor of 20 for the hard-edge lattice solutions. In Fig. 2, we show the results of the emittance reduction in each of the three dimensions of the 4-dipole ring.

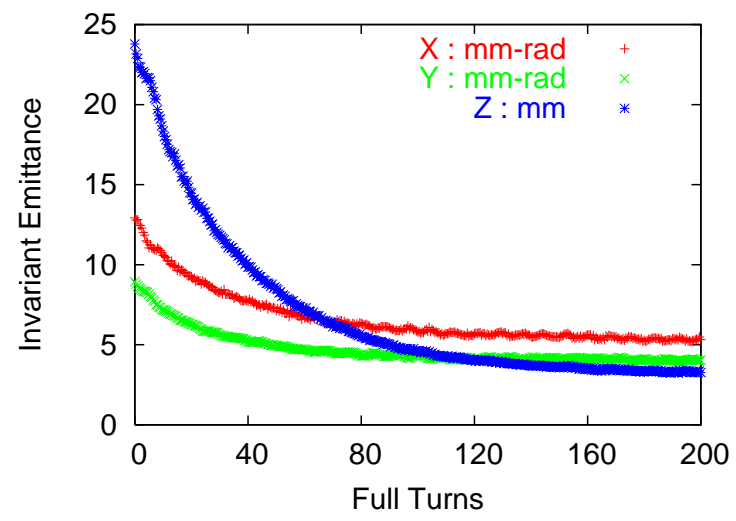

Figure 2: Invariant emittances for each dimension as a function of full turns in the ring.

\section{SOFT-EDGE SOLUTIONS}

We have further explored lattice solutions with softedge Maxwellian magnetic fields. Magnet design and field computations are computed using the finite element code TOSCA[12].

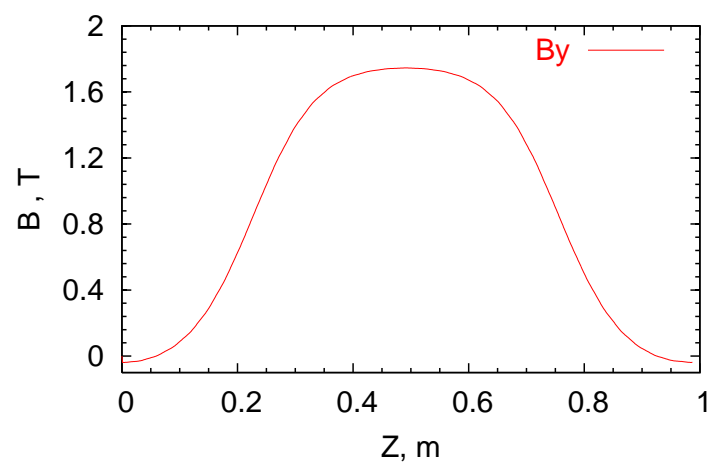

Figure 3: The $\mathrm{B}_{y}$ component of the calculated dipole field.

A single magnet is modeled and the presence of the other magnets is taken into account with the boundary conditions. TOSCA provides the ability to track particles within the program. By launching muons at various start positions on a symmetry plane, a closed orbit can be found. Using a mid-plane field map from TOSCA, the field and its harmonics can be calculated along the closed orbit path. We show in Fig. 3, the longitudinal profile of the dipole field as seen along the closed orbit path of a circulating reference particle with a momentum of $172 \mathrm{MeV} / \mathrm{c}$. Details for this procedure can be found in Ref.[13].
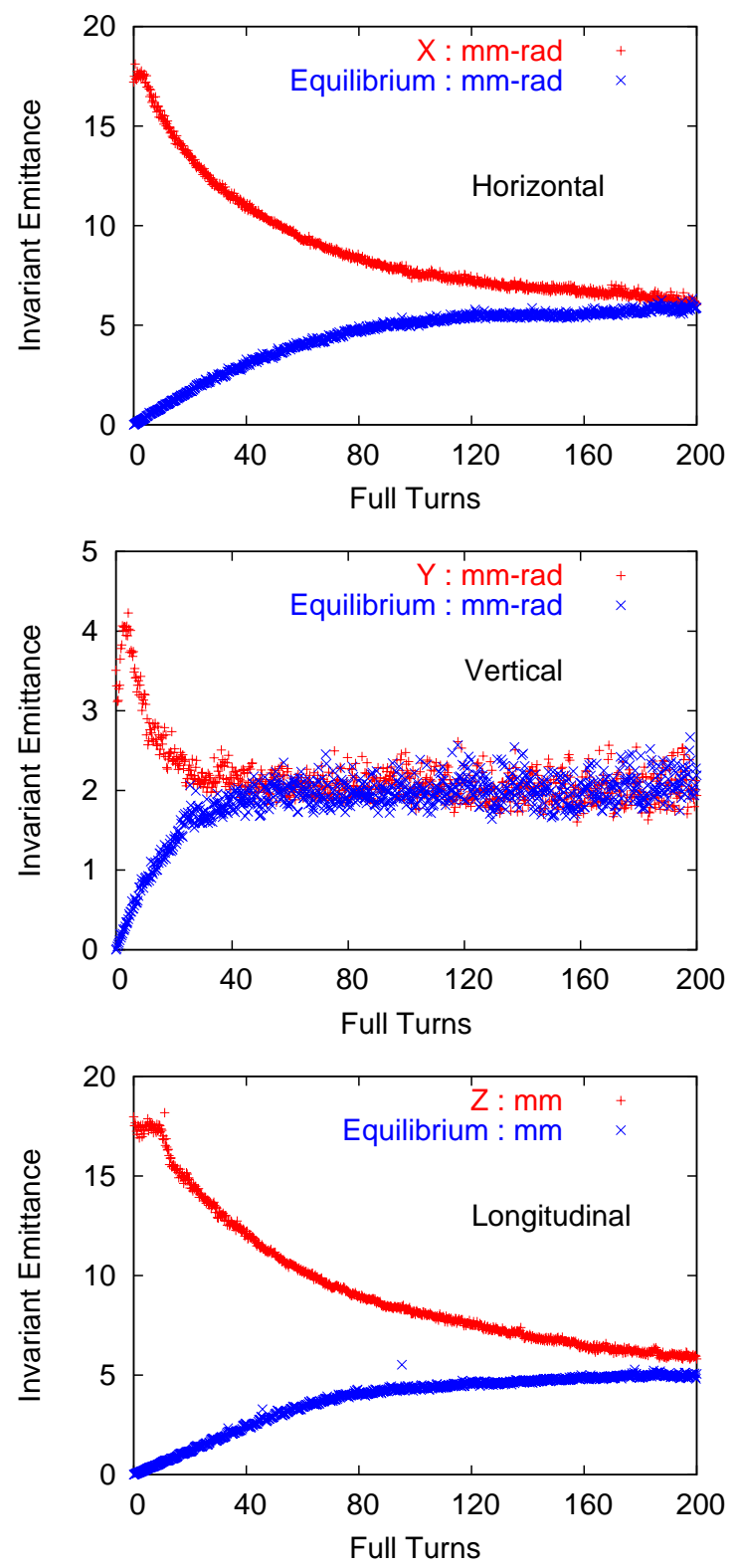

Figure 4: Evolution of invariant emittances for warm and cold muon beams.

The ICOOL simulation program can accept the field as a Fourier decomposition of each of these field harmonics along the closed orbit path. The field can be reconstructed by an expansion in variables of the local coordinate system defined by the closed orbit path.

The input beam admittance for each case is determined 
by first launching a broad spectrum of particles. This broad spectrum beam is allowed to circulate without the influences of multiple Coulomb scattering and energy straggling. Those particles which survive multiple turns are then re-injected with their initial start values and stochastic processes are switched on.

Fig. 4 shows the evolution of the invariant emittances for each dimension for the case of case of operating with a gas pressure of 10 atmospheres at $77^{0} \mathrm{~K}$ (40 atmospheres at room temperature) and a peak axial rf voltage of $10 \mathrm{MV} / \mathrm{m}$. The $\mathrm{rf}$ frequency is $201.25 \mathrm{MHz}$. We follow with interest the development elsewhere of $201.25 \mathrm{MHz}$ pillbox $\mathrm{rf}$ cavities[14] for the acceleration of muon beams.

\section{INJECTION/EJECTION}

For purposes of demonstration, we intend to inject muons into the ring as single particles rather than as a bunch. Our strategy will be to exploit the $\mathrm{dE} / \mathrm{dx}$ energy losses of the loaded high-pressure hydrogen gas which will cause the radius of the circulating particles to be reduced. We will introduce additional absorbers at a high radius which will cause the radius of each particle orbit to be sufficiently reduced so that the supplemental absorbers will not be traversed on subsequent orbits. We note that ejection is not necessary for a proof-of-principle machine but will none-the-less be easier than the injection problem due to the fact that the $6 \mathrm{D}$ phase space of the beam will be greatly reduced.

\section{CONCLUSION}

We have shown that a proof-of-principle cooling ring for muon beams can be realized with modest operational parameters. We show in Fig. 5 a schematic layout of our proposed ring.

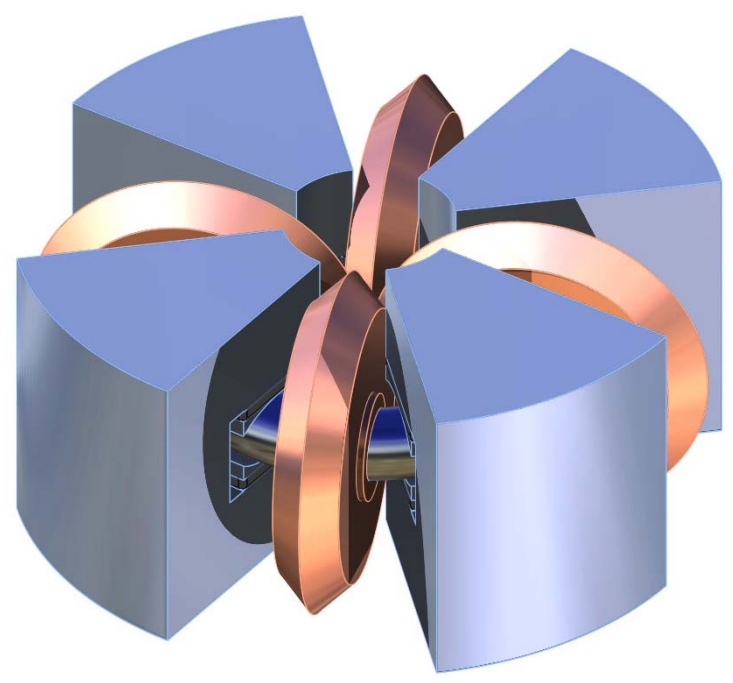

Figure 5: Layout of the cooling ring. The diameter of the cylindrical $201.25 \mathrm{MHz}$ rf cavities is $1.3 \mathrm{~m}$

\section{ACKNOWLEDGMENTS}

This work was performed with the support of the US DOE under Contract NO. DE-AC02-98CH10886 and, in part, by Particle Beam Lasers, Inc. under US DOE SBIR Grant No. DE-FG02-04ER84037.

\section{REFERENCES}

[1] H.G. Kirk, Targetry for a $\mu^{+} \mu^{-}$Collider, Proceedings of the 1999 Particle Accelerator Conference, New York, NY, June 2001, p. 3029.

[2] K.T. McDonald et al., The Primary Target Facility for a Neutrino Factory Based on Muon Beams, Proceedings of the 2001 Particle Accelerator Conference, Chicago, IL, June 2001, p. 1583.

[3] R.B. Palmer, Progress on $\mu^{+} \mu^{-}$Colliders, Proceedings of the 1997 Particle Accelerator Conference, Vancouver, B.C., Canada, May 1997, p. 286.

[4] S. Ozaki, R. Palmer, M. S. Zisman and J. Gallardo, Editors, Feasibility Study-II of a Muon-Based Neutrino Source, BNL-52623 (June 2001); http://www.cap.bnl.gov/mumu/studyii/FS2-report.html.

[5] Juan C. Gallardo et al., An Ionization Cooling Channel for Muon Beams Based on Alternating Solenoids, Proceedings of the 1999 Particle Accelerator Conference, New York, NY, June 2001, p. 3032.

[6] Pierrick M. Hamlet et al., Studies of RF Breakdown of Metals in Dense Gases, Proceedings of the 2005 Particle Accelerator Conference, Knoxville, TN, May 2005 (these proceedings).

[7] A.A. Garren, A.S. Kenney, E.D. Courant, A.D. Russel and M.J. Syphers, SYNCH, A program for Design and Analysis of Synchrotrons and Beamlines, LBL-34668, BNL-49925, FNAL-PUB-94/013 (1993).

[8] R.C Fernow, ICOOL: A Simulation Code for Ionization Cooling of Muon Beams, Proceedings of the 1999 Particle Accelerator Conference, New York, NY, March 1999, p. 3020 .

[9] H. Kirk, D. Cline, Y, Fukui and A. Garren, Progress Toward a Muon Ring Cooler, Accelerator M1 Working Group Report, Snowmass 2001 Workshop, Snowmass, Colorado, 2001; http://www.slac.stanford.edu/econf/C010630/papers/ M101.PDF .

[10] Harold Kirk, David Cline, Yasuo Fukui and A. Garren, Muon Storage Rings for 6D Phase-Space Cooling, Proceedings of the 2003 Particle Accelerator Conference, Portland, OR, May 2003, p. 2008.

[11] D.J Summers et al., 6D Ionization Muon Cooling with Tabletop Rings, Proceedings of the 2004 Annual Meeting of the DPF of the American Physical Society, Riverside, CA, 26-31 Aug. 2004.

[12] The TOSCA program is described in the Opera-3D Reference Manual VF-05-03-B2 from Vector Fields.

[13] S.A. Kahn et al., Design of a Magnet System for a Muon Cooling Ring, Proceedings of the 2005 Particle Accelerator Conference, Knoxville, TN, May 2005 (these proceedings).

[14] A. Ladran et al., Analysis of a Grid Window Structure for RF Cavities in a Muon Cooling Channel, Proceedings of the 2003 Particle Accelerator Conference, Portland, OR, May 2003, p. 2023. 